Pathophysiology of Haemostasis and Thrombosis

\title{
Biochemistry and clinical pharmacology of new anticoagulant agents
}

\author{
Meyer Michel Samama, Grigoris T. Gerotziafas, Ismail Elalamy, Marie Hélène Horellou, \\ Jacqueline Conard
}

Service d'Hématologie Biologique, Hôpital Hôtel-Dieu de Paris, France.

\section{Key Words}

Pentasaccharide, fondaparinux, DX-9065a, melagatran, ximelagatran, hirudin, bivalirudin, argatroban, antithrombin

\section{Summary}

New anticoagulants have been designed using advances in the biotechnology and the biochemistry of the clotting factors. They are classified according to their target or their mechanism of action (AT-dependent or AT-independent). Antithrombotic activity after oral administration is another important property. This abstract is restricted to the synthetic pentasaccharide and the ximelagatran/melagatran.

Fondaparinux (Arixtra ${ }^{\circledR}$ ) is a chemically synthesized methoxy derivative of the natural pentasaccharide ( $\mathrm{MW}=1728 \mathrm{Da}$ ). Fondaparinux has nearly complete bioavailabity after subcutaneous injection. The elimination half-life is about 17 hours. Fondaparinux is cleared from the kidneys. In contrast to heparin, fondaparinux does not interact with plasma proteins other than antithrombin and it does not cross-react in vitro with heparin induced thrombocytopenia antibodies. Fondaparinux inhibits specifically FXa, although some other targets are suspected (FIXa and FVIla).
Endogenous levels of AT are the rate limiting factor for its anti-Xa activity. Fondaparinux, in contrast to direct FXa inhibitors (i.e. DX-9065a) inhibits free FXa but not the FXa bound to prothrombinase, and it does not prolong PT and aPTT. Fondaparinux inhibits thrombin generation in human plasma. We have shown that the inhibition of thrombin generation by fondaparinux is inversely correlated with thromboplastin concentration. This original observation may explain the absence of any effect of fondaparinux on PT.

Melagatran dipeptide ( $M W=430 \mathrm{Da})$ is a specific, reversible direct thrombin inhibitor. It inhibits free and clot bound thrombin. Its half-life is 1.7-2.5 hours after iv or sc administration. Ximelagatran (Exantas ${ }^{\circledR}$ ), is a prodrug with a hydroxyamidino instead of amidino-group and an esterified carboxyl-group. It is the first clinically used direct orally acting thrombin inhibitor. Ximelagatran after intestinal absorption is metabolized to melagatran. Melagatran is not metabolized and is cleared through the kidney. Among the specific FXa inhibitors the synthetic pentasaccharide (FondaparinuxArixtra $\left.{ }^{\circledR}\right)$ has been approved by FDA and several European health organizations for the prophylaxis of VTE in major orthopedic surgery. No specific antidote has been described for the new antithrombotics. Encouraging results have been obtained from our

\section{KARGER \\ (C) 2002 S. Karger AG. Base \\ $1424-8832 / 02 / 0326-0218 \$ 18.50 / 0$}

Professor Emeritus of Hematology, Meyer-Michel Samama, Hôtel-Dieu, 75181 PARIS CEDEX 04, France; phone number: 331423482 66; fax number: 331423482 54; E-Mail: labo.hematologie@htd.ap-hop-paris.fr 
group on the possible use of rFVIla (Novoseven $\AA$ ). The general opinion is that there is no need for laboratory monitoring of the new antithrombotic agents. Conclusion. The research for safer and more effective anticoagulants has been successful by targeting specific steps in coagulation. These single-targeted agents are challenging multi-targeted drugs (heparins and vitamin-K antagonists). Other drugs including orally active FXa direct inhibitors will probably enrich the armentarium of antithrombotic drugs.

Copyright ( 2002 S. Karger AG. Basel

\section{Introduction}

Besides anti-platelet therapy, antithrombotic therapy is currently based on three classes of agents, i.e. unfractionated heparin (UFH), low-molecular-weight heparins (LWMHs) and vitamin $\mathrm{K}$ antagonists (VKA). Both heparins and oral anticoagulants (vitamin $\mathrm{K}$ antagonists) are efficacious antithrombotic drugs but their use has well-known limitations. They are not selective, acting on a broad range of substrates in the coagulation cascade. In addition, heparins are a heterogeneous mixture of different molecules purified from animal tissues and with variable antithrombotic activity.

New anticoagulant agents have been designed taking into consideration recent advances in the biotechnology, in the knowledge of the mechanisms of blood coagulation and the biochemistry of the structure and function of the clotting factors as well as the observations on the blood sucking organisms such as leech, tick and ankylostoma which in their saliva possess substances with very potent anticoagulant activity.

According to their target the new anticoagulants are grouped into three categories:

1. Agents that block the first stages of blood coagulation involving tissue factor and factor VIIa or IXa.

2. Agents that block later targets in the coagulation process by inhibiting factor $\mathrm{Xa}$ and thus down-regulate both thrombin generation and feedback FXa mediated activation loops, which lead to prothrombinase formation.

3. Agents that inhibit thrombin.

According to their mechanism of action the new anticoagulants are grouped into two categories:

1. The indirect inhibitors, which act by enhancing the inhibitory activity of AT. Such inhibitors are heparins (UFH and LMWHs) and the synthetic pentasaccharide.

2. The direct inhibitors, which act directly on the target serine protease. Some examples of such inhibitors are hirudin, hirulog, argatroban, ximelagatran/melagatran and DX-9065a

\section{Inhibitors of TF pathway and intrinsic tenase}

Among the antithrombotic agents aiming the tissue factor (TF) pathway, the active site inhibited factor VIIa (ASIS) is in the most advanced stage of research. Recombinant FVIIa lacking the Gla-domain, is converted into a catalytically inert protein by reactive site residues reacting with Phe-Phe-Argchloromethyl ketone. ASIS retains its affinity for TF and competes for FVIIa and FVII binding to TF, blocking FVIIa activity and FVII to FVIIa activation [1]. Another approach is the production of anti-TF monoclonal antibodies against specific epitopes on TF, which block the formation of TF/FVIIa complex resulting in a significant antithrombotic effect [2].

Inhibitors of TF/FVIIa enzymatic activity are also being developed. Among these, recombinant TFPI, the natural inhibitor of TF pathway, has been shown to be effective in prevention of thrombosis in sepsis and disseminated intravascular coagulation models. Nematode anticoagulant protein c2 (NAPc2) is a potent selective inhibitor of the TF/FVIIa complex in the presence of FXa, which is present in the saliva gland of the hookworm Ancylostoma caninum.

\section{Inhibitors of factor $\mathrm{Xa}$}

Among the specific FXa inhibitors the synthetic pentasaccharide (Fondaparinux, Arixtra ${ }^{\circledR}$ ) is in the most advanced stage of clinical development and has been approved by FDA and several European health organizations for the prophylaxis of VTE in major orthopedic surgery.

The direct factor Xa inhibitor DX-9065a is actually being studied in phase II clinical trials.

\section{Indirect (AT-dependent) factor Xa inhibitors}

Fondaparinux is a chemically synthesized methoxy derivative of the natural pentasaccharide sequence, which is the active site of heparin chains that mediates the interaction with antithrombin. It has a molecular weight of 1728 Daltons. It has been obtained by chemical synthesis including a large number (about 50) of steps (Figure 1). Contrary to heparins purified from animal tissues, this molecule does not exist as such in nature. The risk of contamination with animal pathogens is therefore eliminated and the batch-to-batch consistency is very high. In addition, since fondaparinux represents a single molecular entity with a well-defined pharmacological target, the plasma concentration of the product can be expressed on a gravimetric basis $(\mu \mathrm{g} / \mathrm{mL}$ or $\mu \mathrm{mol} / \mathrm{L})$ and not in international units like heparins.

Mechanism of action of fondaparinux. Fondaparinux binds to a specific site on antithrombin with high affinity (dissocia- 
tion constant, $\mathrm{Kd}=36 \pm 11 \mathrm{nM}$ ) and in a 1:1 stoichiometric relationship. Fondaparinux increases by about 300 -fold the rate of factor Xa inhibition by antithrombin. Whereas the interaction of antithrombin with factor $\mathrm{Xa}$ is irreversible, that of fondaparinux with antithrombin is reversible. Thus, each molecule of fondaparinux can bind to and activate several molecules of antithrombin in succession. Fondaparinux occupies only a part of the heparin-binding site on antithrombin and the conformational change induced by the binding of fondaparinux to antithrombin differs from that induced by heparin. In contrast to heparin, pentasaccharide does not induce TFPI release and it does not modify the activity of TAFI (for relevant review see [3]). Fondaparinux acts solely by the antithrombinmediated inhibition of factor Xa [4]. However, some other targets may be suspected, such as inhibition of factor IXa [5] and antithrombin mediated inhibition of factor VIIa generation and activity [6,7]. Endogenous levels of AT are the rate limiting factor for antithrombotic anti-Xa activity of fondaparinux. Once antithrombin is saturated, addition of more molecules of fondaparinux has no further anticoagulant effect [8].

By inhibiting thrombin generation, but not thrombin activity, fondaparinux therefore maintains thrombin-mediated physiological loops. Fondaparinux in contrast to direct FXa inhibitors (i.e. DX-9065a) inhibits free factor Xa but not factor $\mathrm{Xa}$ bound to prothrombinase complex (FXa, FVa phospholipids and calcium ions) [9]. Moreover, fondaparinux inhibits factor Xa bound to fibrin network (the IC50 is about $0.8 \mu \mathrm{g} / \mathrm{ml}$ to $1.5 \mu \mathrm{g} / \mathrm{ml}$ ) but it does not have any effect on fibrin bound prothrombinase [10].

Fondaparinux has a significant antithrombotic effect in prevention and treatment of venous thromboembolism and it inhibits thrombin generation after triggering either tissue factor or intrinsic clotting pathway. However, it has no significant effect on prothrombin time (PT) and activated partial thromboplastin time (aPTT). Interestingly on the other hand fondaparinux prolongs the Heptest. We have recently shown that the inhibition of thrombin generation induced by therapeutic concentrations of fondaparinux is inversely correlated with the concentration of thromboplastin, whereas it has no effect on thrombin generation when a crude solution of thromboplastin is used. This original observation may bring an explanation for the absence of any effect of fondaparinux on PT since an excess concentration of thromboplastin (a mixture of tissue factor and phospholipids) is added in PT assay [11].

Pharmacokinetics of fondaparinux The pharmacokinetics of fondaparinux appears predictable and consistent, making routine monitoring and dose adjustments unnecessary. Furthermore, the non-specific binding of heparins to platelet factor 4 may explain both their inefficacy with respect to platelet-rich arterial thrombosis, and the occurrence of heparin-induced thrombocytopenia (HIT). Regarding this latter side effect, fondaparinux did not cross-react with any sera from 25 patients with heparin-induced thrombocytopenia [12].
The pharmacokinetic parameters of fondaparinux were determined in young and elderly healthy human volunteers [13]. The peak plasma level (Cmax) was obtained two hours after the subcutaneous injection. The elimination half-life was dose-independent and ranged from 13 to 20 hours, which allows a convenient once-daily dosing regimen. In elderly subjects, who had a lower creatinine clearance than younger ones, the elimination half-life of fondaparinux was slightly increased.

The steady state plasma concentrations were reached after two or three days of repeated subcutaneous administration of escalating doses of fondaparinux (6 to $18 \mathrm{mg}$ ) to elderly healthy volunteers for seven consecutive days. Peak plasma levels were 1.5 to 2 times higher than those generated after the first injection. The kidneys were the major route of elimination and at steady state, the fraction of the injected fondaparinux dose found in the urine ranged between $53 \%$ and $84 \%$. At increasing doses of fondaparinux a plateau is reached once AT has been saturated and free fondaparinux is rapidly eliminated through the kidney.

The "meta-pentasaccharides". Chemical modifications of the original synthetic pentasaccharide aimed to the improvement of its pharmacokinetics and pharmacodynamics by increasing the affinity to AT. The principal modification of the original pentasaccharide consists in the replacement of N-sulfated groups from sulfated esters. Moreover, the methylation of hydroxylated groups further increases the affinity for antithrombin. A pharmacokinetic study including 15 structural analogues of fondaparinux showed a close relationship between the affinity for AT of these compounds and their halflife in rats [14].

Idraparinux has been selected for phase II clinical trials.

\section{Direct (AT independent) factor $X a$ inhibitors}

Tick anticoagulant peptide, (TAP) and antistasine (ATS) are among the best studied natural FXa inhibitors. TAP, originally purified from soft tick Ornithodoros moubata, has been fully characterized as a reversible competitive, slow binding inhibitor of FXa. ATS is a competitive, slow-tigh binding inhibitor that has been purified from the salivary glands of the Mexican leech Haementeria Officinalis. Studies in animal models of thrombosis showed that both direct specific FXa inhibitors provide equal or superior efficacy to that achieved by heparin or the inhibition of thrombin directly. The advantages in this area prompted the research for small molecules, synthetic direct inhibitors of FXa with potential therapeutic efficacy. Among several such agents (i.e. PRP 130737, YM-60828, YM-75466, PRP 120844, SEL-2711, BX-807834) the DX9065a is in the most advanced stage of clinical development.

DX-9065a is the first of a new class of small-molecule orally active direct FXa inhibitors. DX-9065a is a synthetic 
propanoic acid (Figure 1) derivative with a molecular weigh of 571 Daltons [15]. It specifically neutralizes factor $\mathrm{Xa}$ $(\mathrm{Ki}=0.041 \mu \mathrm{mol} / \mathrm{l})$ and exerts little effect on other proteases, particularly thrombin $(\mathrm{Ki}>2000 \mu \mathrm{mol} / \mathrm{l})$. DX-9065a inhibits free factor $\mathrm{Xa}$ and factor Xa bound to prothrombinase [10]. Therapeutic concentrations of DX-9065a induce prolongation of thrombin generation lag-time and inhibition of endogenous thrombin potential, in in vitro thrombin generation induced by activation of TF-pathway in human plasma [16]. Moreover it efficiently inhibits clot-bound prothrombinase, at concentrations as low as $15 \mathrm{ng} / \mathrm{ml}$ [10]. DX-9065a prolongs PT and aPTT but surprisingly does not prolong the Heptest. Its antithrombotic efficacy has been shown in animal models of disseminated intravascular coagulation, venous thrombosis and vein graft thrombosis.

DX-9065a exhibits renal clearance and after a single increasing, intravenous doses of DX-9065a, peak plasma concentrations reached $1640 \mathrm{ng} / \mathrm{ml}$ in subjects receiving $30 \mathrm{mg}$ over a period of 1 hour [17]. After a 72 hours intravenous infusion of DX-9065a, at doses escalating from $15 \mathrm{ng} / \mathrm{ml}$ to 200 $\mathrm{ng} / \mathrm{ml}$, in patients with stable coronary disease, plasma drug levels correlated strongly with plasma anti-Xa activity and prothrombin time prolongation, but less so with aPTT [18].

\section{Thrombin inhibitors}

\section{Indirect (AT-dependent) thrombin inhibitors}

The heparinomimetics. Considering compounds with the same antithrombin-binding domain (pentasaccharide domain), an increase in the number of charges results in a decrease of the corresponding half-lives of these compounds in plasma. It was shown that synthetic oligosaccharides with 15,17 or 19 units, possessing the antithrombin binding pentasaccharide) were capable to catalyze the antithrombin dependent inhibition of both FXa and thrombin but their activity was neutralized by PF4. Therefore, the addition of an uncharged spacer in the structure of the molecule (which reduced the global charge of the compound and allowed the formation of a "charge cluster" below a critical size) allowed to obtain compounds with pharmacokinetics close to those of its AT binding domain and with clear renal mode of elimination [19]. Clinical trials will show whether such synthetic heparinomimetics can replace heparin, which is more than half a century old.

\section{Direct (AT-independent) thrombin inhibitors}

Direct synthetic and selective thrombin inhibitors; including hirudin, hirulog, argatroban, and melagatran have been developed [20]. A few oral direct thrombin inhibitors are being developed. An important advance in the development of thrombin inhibitors is the discovery of Ximelagatran (H376/95) an oral prodrug of Melagatran.

Hirudin, exists in the saliva of the leech. Recombinant hirudin is in the most advanced stage of clinical development. Hirudin is a 65 amino acids protein with a molecular weight is 6975 Daltons, originally extracted from the salivary gland of the medicinal leech (Hirudo medicinalis). Hirudin is a direct (AT-independent), specific and irreversible stoichiometric inhibitor of thrombin. Activity of recombinant hirudin is close to the naturally occurring hirudin. The carboxy-terminal domain of hirudin binds to the substrate binding exosite I of thrombin and the amino-terminal domain of hirudin binds to the active site of thrombin [21]. The dissociation constant is extremely low $\left(\mathrm{Ki}=10^{-12}\right.$ to $\left.10^{-14} \mathrm{M}\right)$. Due to its high affinity for thrombin, hirudin inhibits almost all the physiological actions of thrombin. Hirudin does not bind to antithrombin, it is not neutralized by platelet factor 4 or any other protein released by the activated platelets and it does not cross-react in vitro with the antibodies from patients with heparin induced thrombocytopenia (HIT). Recombinant hirudin induces prolongation of PT, aPTT, thrombin clotting time and ecarin clotting time. The later test is the most mandatory for laboratory monitoring of treatment with rhirudin. Hirudin in contrast to heparin inhibits both free and clot bound thrombin.

After intravenous administration the plasma half-life of $r$ hirudin is about 40 minutes and 120 min after subcutaneous administration. Recombinant hirudin is eliminated principally from the kidneys. Recombinant hirudin appears to have a fairly narrow therapeutic window and this has been a relative limitation to its use. The r-hirudin (Lepirudin, Refludan ${ }^{\circledR}$ ) has been approved for the treatment of HIT by the European Health Organization and the FDA in the USA. A pegylated form of r-hirudin, with a longer half-life, is being developed.

Bivalirudin is a synthetic bivalent thrombin inhibitor. It is a dodecapeptide analogue of the carboxy-terminal domain of hirudin that binds to the substrate binding exosite I of thrombin, linked to the active site moiety (D-Phe-Pro-Arg-Pro) by 4 glycine residues. In contrast to hirudin, bivalirudin is a reversible inhibitor of thrombin, because once bound to thrombin, the Arg-Pro bond of the amino-terminal domain of bivalirudin is cleaved converting it to a lower affinity inhibitor [22]. Bivalirudin has a shorter half-life than rhirudin, which may render it safer.

Argatroban is a carboxylic acid derivative, which binds non covalently to the active site of thrombin. Argatroban has been approved by FDA in USA for the treatment of HIT.

Melagatran. The discovery of a series of molecules capable of inhibiting thrombin and their structure was derived from the site of the fibrinogen molecule to which binds the active site of thrombin [23]. Thrombin cleaves the peptide bonds Arg16/Gly17 and Arg19/Val20 of the chain A $\alpha$ of fibrinogen. Melagatran, which is a dipeptide (Figure 1) with a molecular weight of 430 Daltons mimics this domain of fibrinogen and working as a "trap" binds to the active site of thrombin. It is a specific, reversible thrombin inhibitor $(\mathrm{Ki}=2 \mathrm{nM})$. Melagatran does not neutralize all the physiological functions of thrombin. 
Recombinant hirudin, melagatran and other direct thrombin inhibitors are much more effective than heparin-AT in inhibiting both free and fibrin bound thrombin, which may be an important property that may reduce the re-occlusion after coronary thrombolysis and the propagation of thrombosis in DVT. Clinical pharmacology has clearly shown that the activity on free and clot bound thrombin and the ratio of the IC50 determined for the two activities could be an important characteristic of these two inhibitors.

Specific thrombin inhibitors such as hirudin and melagatran are generally more active against thrombin than on prothrombin activation and thrombin generation [24].

Melagatran has a short half-life of 1.7 hours after intravenous administration. Melagatran is not metabolized and is excreted unmodified through the kidney. After oral administration of melagatran, its bioavailability is very low. A chemical modification of the two extremities of the molecule succeeded in producing the ximelagatran (Exantas $\left.{ }^{\circledR}\right)$ a prodrug, which has a bioavailability around $20 \%$ after oral administration [25]. Two different metabolites have been identified. The peack concentration of melagatran is obtained 2 hours after
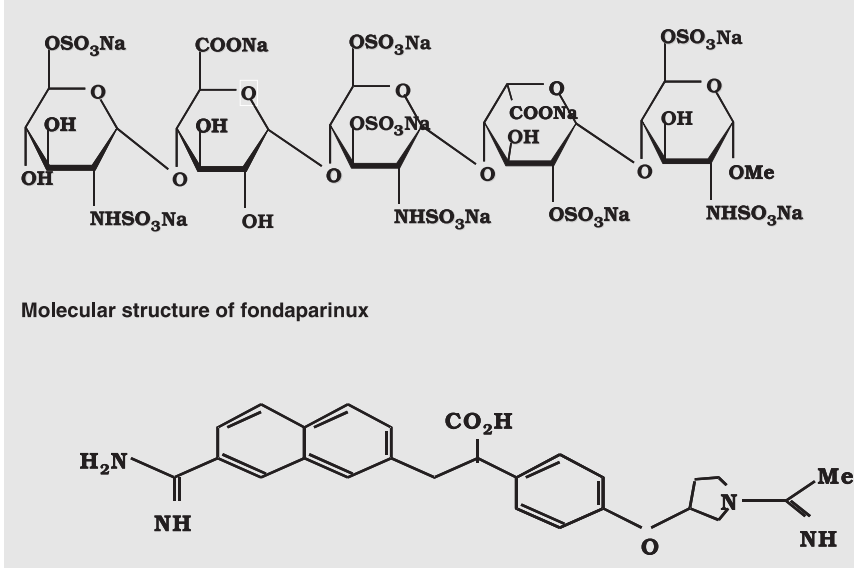

Molecular structure of DX-9065a

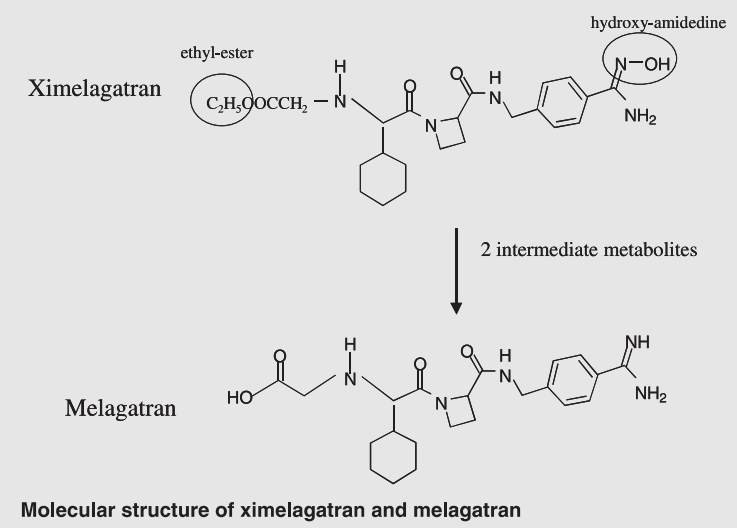

Fig. 1. Molecular structures of synthetic pentasaccharide (Fondaparinux), DX-9065a, and ximelagatran/melagatran. ingestion of ximelagatran. Melagatran is then cleared in less than 12 hours. Due to its antithrombin activity, melagatran induces a prolongation of PT, aPTT, thrombin clotting time and ecarin clotting time. Melagatran and its orally active prodrug Ximelagatran have been studied in several clinical trials in orthopedic surgery. Ximelagatran could be a good candidate for vitamin $\mathrm{K}$ antagonist's replacement.

\section{New second generation of LMWHs}

LMWHs are obtained from different preparations of standard heparin according to divers procedures. Thus, they are different in their biochemical and pharmacological properties and they have different pharmacokinetics. These differences may influence the clinical profile of LMWHs. A greater understanding of structure - activity relationships has led to further modifications in manufacturing process resulting in a second generation of LMWH with a lower mean molecular weight and a more precisely defined composition of polysaccharide chains Bemiparin represents such a second generation LMWHs. Bemiparin has a molecular weight of 3600 daltons and an anti$\mathrm{Xa} /$ anti-IIa ratio higher than 8 [26]. The efficacy and safety of bemiparin in the prevention and treatment of venous thromboembolic disease is being evaluated [27].

\section{Specific aspects of the new anticoagulants}

\section{Antidote}

For almost all of the emerging new antithrombotic agents no specific antidote is available. The need for antidote or for a rapid and safe haemostatic method is more urgent in the case of fondaparinux, which is actually being introduced in the daily clinical practice. An attractive alternative could be the use of rFVIIa (Novoseven ${ }^{\circledR}$ ), which has been show to be an effective and safe haemostatic agent in a variety of clinical conditions without inducing increased thrombin generation [28]. Recent in vitro experiments from our group showed that rFVIIa does not modify the inhibition of the endogenous thrombin potential induced by therapeutic concentrations of fondaparinux, but it significantly accelerates the generation of thrombin [11]. However this possible alternative requires extensive study and cost/effective assessment.

\section{Laboratory monitoring}

The need for laboratory monitoring, of the new antithrombotic agents is an open issue. Based on the results of largescale phase III trials, laboratory monitoring of fondaparinux does not seem necessary for the prevention of venous thromboembolism in major orthopaedic surgery. No data are actually available for DX9065a and ximelagatran. However the need for laboratory monitoring of the new class of specific antithrombotic agents raises several methodological problems. Its clinical relevance requires further study. 


\section{Conclusion}

The research for safer and more effective anticoagulants has been very successful by targeting specific steps in the clotting mechanism. These new agents are challenging over multitargeted drugs such as heparin and vitamin $\mathrm{K}$ antagonists. Fondaparinux is a new synthetic antithrombotic agent developed according to a process of rational drug design. It has been classified by the World Health Organization in the class of "Other antithrombotics" (ATC code: B01AX05), differing notably from UFH and LMWH. Indeed, this chemically synthesized molecule is original with regard to its selective target (factor Xa) and its mode of action (via antithrombin). It has a favorable pharmacokinetic profile allowing a convenient oncedaily administration without routine monitoring of anticoagulant activity or dose adjustments. A new generation of meta- pentasaccharides and heparinomimetics with improved pharmacodynamics and pharmacokinetics is under development. Moreover the results of clinical trials demonstrate that a pure factor Xa inhibitor devoid of antithrombin activity can exhibit a powerful antithrombotic activity in man.

The ximelagatran is the first orally active direct synthetic thrombin inhibitor with favorable pharmacokinetics and pharmacodynamics and clinical efficacy and safety in prevention of thrombosis in major orthopedic surgery. Its efficacy and safety versus the established treatment with vitamin $\mathrm{K}$ antagonists in the prevention of stroke in patients suffering from atrial fibrillation is being investigated.

The forthcoming specific inhibitors are going to enrich the armentarium against the thromboembolic disease, and to raise new questions about the socio-economic impact of the new antithrombotic strategies.

\section{References}

1 Johnson DJ, Nugent PG, Tuddenham EG, Harlos K, Kemball-Cook G Crystallization and preliminary $\mathrm{X}$-ray analysis of active siteinhibited human coagulation factor VIIa (desGla). J Struct Biol 1999;125:90-93.

2 Kirchhofer D, Moran P, Chiang N, Kim J, Riederer MA, Eigenbrot C, Kelley RF. Epitope location on tissue factor determines the anticoagulant potency of monoclonal anti-tissue factor antibodies. Thromb Haemost 2000;84:1072-81.

3 Walenga JM, Jeske WP, Samama MM, Frapaise FX, Bick RL, and Fareed J. Fondaparinux: a synthetic heparin pentasaccharide as a new antithrombotic agent. Expert Opin Investig. Drugs 2002; 11:397-407.

4 Walenga JM, Petitou M, Lormeau JC, Samama M, Fareed J, and Choay J. Antithrombotic activity of a synthetic heparin pentasaccharide in a rabbit stasis thrombosis model using different thrombogenic challenges. Thromb Res. 1987;46:187-98.

5 Wiebe E, O'Brien L, Stafford A, Fredenburgh J, Weitz J. Heparin catalysis of factor IXa inhibition by antithrombin: pentasaccharide induced conformational change in antithrombin predominated over the template effect. Thromb Haemost 2001; Abstract: P2101

6 Lormeau JC, Herault JP, Herbert JM. Antithrombin-mediated inhibition of factor VIIa-tissue factor complex by the synthetic pentasaccharide representing the heparin binding site to antithrombin. Thrombos Haemost 1996; 76:5-8.

7 Gerotziafas GT, Elalamy I, Chakroun T, Bara L, Samama MM. On the mechanism of inhibition of tissue factor pathway by the synthetic pentasaccharide during coagulation of human plasma. Blood Coag Fibrinol in press 2003.

8 Walenga JM, Hoppensteadt D, Mayuga M, Samama MM, Fareed J. Functionality of pentasaccharide depends on endogenous antithrombin levels. Submitted 2002.

9 Hemker HC, Choay J, Beguin S. Free factor $\mathrm{Xa}$ is on the main pathway of thrombin generation in clotting plasma. Biochimica et
Biophysica Acta 1989; 992: 409-411.

10 Herault JP, Bernat A, Pflieger AM, Lormeau JC, Herbert JM.Comparative effects of two direct and indirect factor $\mathrm{Xa}$ inhibitors on free and clot-bound prothrombinase. J Pharmacol Exp Ther 1997;283:16-22.

11 Gerotziafas GT, Depasse F, Elalamy I, Chakroun T, Samama MM. In vitro assessment of fondaparinux antithrombotic potential and influence of rFVIIa (Novoseven) on thrombin generation. 44th American Society of Hematology Annual Meeting, Pennsylvania, USA 2002.

12 Elalamy I, Lecrubier C, Potevin F, Abdelouahed M, Bara L, Marie JP, and Samama M. Absence of in vitro cross-reaction of pentasaccharide with the plasma heparin dependent factor of twenty-five patients with heparin associated thrombocytopenia. Thromb Haemost 1995;74:1384-5.

13 Boneu B, Necciari J, Cariou R, Sié P, Gabaig AM, Kieffer G, Dickinson J, Lamond G, Moelker $H$, Mant $T$, and Magnani $H$. Pharmacokinetics and tolerance of the natural pentasaccharide (SR90107/ORG31540) with high affinity to antithrombin III in man. Thromb Haemost 1995;74:1468-73

14 Van Amsterdam R, Vogel G, Visser A, Kop WJ, Buiting MT, Meuleman DG. Synthetic analogues of the antithrombin III-binding pentasaccharide sequence of heparin. Prediction of in vitro residence time. Arter Thromb and Vasc Biol 1995; 15: 495-503.

15 Hara T, Yokoyoma A, Ishihara H, Yokoyoma Y, Nagahara T, Iwamoto M. DX-9065a, a new synthetic, potent anticoagulant and selective inhibitor of factor Xa. Thromb Haemost 1994;71:314-319.

16 Prasa D, Svendsen L, Sturzebecher J. Inhibition of thrombin generation in plasma by inhibitors of factor Xa. Thromb Haemost 1997;78:1215-1220.

17 Murayama N, McMahon H, Young CG, McCracken NW, Okamura Y, Hakusui $\mathrm{H}$, Tanaka M. Pharmacokinetics of the anticoagulant 14C-DX-9065a in the healthy male volun- teer after a single intravenous dose. Xenobiotica 2000; 30:515-521.

18 Dyke CK, Becker RC, Leiman NS, Hochman J, Bovill EG, Lincoff M, Gerstenblith G, Dzavik V, Gardner LH, Hasselblad V, Zillman LA, Shimoto Y, Robertson TL, Kunitada S, Armstrong PW, Harrington RA. First experience with direct factor $\mathrm{Xa}$ inhibition in patients with stable coronary disease. A pharmacokinetics and pharmacodynamic evaluation. Circulation 2002; 105:2385-2391.

19 Petitou M, Herault JP, Bernat A, Driguez PA, Duchaussoy P, Lormeau JC,Herbert JM. Synthesis of thrombin inhibiting heparin mimetics without side effects. Nature 1999; 398: 417-22.

20 Weitz JI, and Hirsh J. New anticoagulant drugs. Chest 2001;119:95S-107S.

21 Rydel TJ, Ravichandran KG, Tulinsky A, Bode W, Huber R, Roitsch C, Fenton JW. The structure of a complex of recombinant hirudin and human thrombin. Science 1990; 249:277280.

22 Maraganore JM, Bourdon P, Jablonski J, Ramachandran KL, Fenton JW. Design and characterisation of hirulogs : a novel class of bivalent peptide inhibitors of thrombin. Biochemistry 1990;29:7095-7101.

23 Gustafsson D, Nyström JE, Carlsson S et al. The direct thrombin inhibitor melagatran and its oral prodrug $\mathrm{H} 376 / 95$ : intestinal absorption properties, biochemical and pharmacodynamic effect. Thromb Res 2001;101:171-18

24 Gerotziafas GT, Bara L, Bloch MF, Makris PE, Samama MM. Comparative effects of synthetic pentasaccharide, low molecular weight heparin, unfractionated heparin and recombinant hirudin on the generation of factor VIIa and prothrombin activation after coagulation of human plasma. Blood Coagulation and Fibrinolysis 1998;9: 571-580.

25 Gustafsson D, Nyström JE, Carlsson S et al. The direct thrombin inhibitor Melagatran and its oral prodrug $\mathrm{H}$ 376/95: intestinal absorption properties, biochemical and pharmacodynamic effect. Thromb Res 2001;101:171-181 
26 Falkon L, Saenz - Campos D, Antonijoan R, Martin S, Barbanoj M, Fontcuberta J. Bioavailability and pharmacokinetics of a new low molecular weight heparin (RO-11). A three way cross-over study in healthy volunteers. Thromb Res 1995; 78:77-86.

27 Navarro-Quilis A, Castellet E, Rocha E, PazHimenez J, Planes A. Efficacy and safety of bemiparin compared with enoxaparin in the prévention of venous thromboembolism after total knee arthroplasty : a randomized doubleblind clinical trial. J Throm Haemost 2002;1:425-432.

28 Gerotziafas GT, Zervas K, Arzoglou P, Karavaggeli E, Parashou S, Van Dreden P, Christakis J, Samama MM. On the mechanism of action of rFVIIa administered to patients with severe thrombocytopenia and life-threatening haemorrhage. Focus on prothrombin activation. British Journal of Hematology 2002; 117:705-708. 